\title{
Tests of human olfactory function: Principal components analysis suggests that most measure a common source of variance
}

\author{
RICHARD L. DOTY, RICHARD SMITH, DONALD A. MCKEOWN, and JAYA RAJ \\ University of Pennsylvania Medical Center, Philadelphia, Pennsylvania
}

\begin{abstract}
It is not known whether nominally different olfactory tests actually measure dissimilar perceptual attributes. In this study, we administered nine olfactory tests, including tests of odor identification, discrimination, detection, memory, and suprathreshold intensity and pleasantness perception, to 97 healthy subjects. A principal components analysis performed on the intercorrelation matrix revealed four meaningful components. The first was comprised of strong primary loadings from most of the olfactory test measures, whereas the second was comprised of primary loadings from intensity ratings given to a set of suprathreshold odorant concentrations. The third and fourth components had primary loadings that reflected, respectively, mean suprathreshold pleasantness ratings and a response bias measure derived from a yes/no odor identification signal detection task. In an effort to adjust for potential confounding influences of age, gender, smoking, and years of schooling on the component structure, a matrix of residuals from a multiple regression analysis, which included these variables, was also analyzed. A similar component pattern emerged. Overall, these findings suggest, in healthy subjects spanning a wide age range, that (1) a number of nominally distinct tests of olfactory function are measuring a common source of variance, and (2) some suprathreshold odor intensity and pleasantness rating tests may be measuring sources of variance different from this common source.
\end{abstract}

Since the pioneering work of Valentin (1848), who determined the lowest concentration of an odorous gas that a subject could perceive, a plethora of nominally distinct olfactory tests has been developed, including tests of sensitivity (e.g., odor detection and recognition thresholds), discrimination, identification, memory, and suprathreshold intensity (for reviews, see Cain, 1978; Doty, 1991, 1992; Engen, 1982; Köster, 1975; Takagi, 1989; Wenzel, 1948). Implicit in the development and application of such tests are the assumptions that they (1) measure specific sensory attributes associated with their names and (2) depend largely upon independent higher order neural substrates. For example, tests of odor memory are assumed to measure the ability to remember odors and to activate cortical circuits related to odor memory processing. Likewise, tests of odor identification are assumed to measure the ability to identify odors and to activate neural circuits associated with odor identification.

\footnotetext{
This work was supported by Grant PO1 DC 00161 from the National Institute on Deafness and Other Communication Disorders and Grant AG 08148 from the National Institute on Aging. We thank David Hildebrand, Donald Morrison, David Smith, Paul Shaman, and Richard Frye for help and advice in setting up the statistical procedures and approaches used in this work. Address correspondence to R. L. Doty, Director, Smell and Taste Center, Hospital of the University of Pennsylvania, 3400 Spruce St., Philadelphia, PA 19104.
}

Despite the intuitively compelling nature and widespread tacit acceptance of these assumptions, little is known about the degree to which different olfactory tests measure distinct perceptual attributes or unique neural substrates. Importantly, one can envision situations in which the logic of such assumptions breaks down and misleading inferences result from literal interpretations of test results. For example, if one has little ability to detect or recognize odorants, then poor performance would be present on an odor memory test, even though the underlying neural circuits for odor memory, per se, may be intact. In this case, the "odor memory" score is not a valid reflection of the substrate of odor memory, since no olfactory sensation to be remembered is available for encoding.

The question of what various olfactory tests actually measure brings to mind the early days of test measurement theory, when psychological tests were initially classified under categories assumed to assess separate mental functions (e.g., Whipple, 1914). Such classification, however, was found to be inaccurate. As may be the case with olfactory tests,

Most tests of mental ability exhibit some degree of positive correlation; often two tests that are classified under the same name exhibit no more correlation than do two other tests supposedly belonging to two different categories of ability. The notion of broad unitary powers which operate singly and in an isolated manner must therefore be discarded. (Guilford, 1954, p. 471) 
The purpose of the present study was to determine, in healthy subjects spanning a wide age range, the degree to which a number of nominally distinct olfactory tests are related to one another. Although several earlier studies have noted correlations between tests of odor detection and identification (e.g., Cain \& Rabin, 1989; Doty, Shaman, \& Dann, 1984), as well as among threshold tests incorporating disparate odorants (Yoshida, 1984), no study of the relationships among a wide variety of olfactory tests has been performed. In the present work, common and independent sources of variance among the tests were explored by using principal components analysis, which, unlike factor analysis, provides determinant weighted composites of the variables under study (for a discussion of this issue, see Rozeboom, 1982; Wilkinson, 1990).

\section{Method}

Subjects. Ninety-seven healthy subjects $(37$ men, 60 women: mean age $=45.84, S D=20.17$; mean years of education $=15.38$, $S D=2.04$; number of current, past, and nonsmokers $=12,36,49$, respectively) participated. All scored well on the 40-item Picture Identification Test (PIT), a test designed to detect individuals with cognitive deficits that would interfere with nonolfactory components of the University of Pennsylvania Smell Identification Test (UPSIT; see below) (mean PIT $=39.90, S D=0.42$; see Vollmecke \& Doty, 1985). The subjects were students and staff of the University of Pennsylvania and active, ambulatory participants recruited from the Philadelphia Center for Older People and the Medford Leas retirement community (Medford, NJ). Each subject received $\$ 25$ for participation and provided informed written consent, in accord with the policies of the University of Pennsylvania's Committee on Studies Involving Human Beings.

\section{Test Procedures}

A battery of nine tests of olfactory function was administered to each subject; presentation order was random. These tests, described in detail below, were chosen to represent a broad range of types of nominally distinct olfactory tests, including tests of odor identification, discrimination, detection, memory, and suprathreshold intensity and pleasantness perception. Several of these tests (Numbers 1, 2, 3, 4, and 9) are widely used clinically and were chosen not only because of this fact, but because they nominally represent tests of odor identification as well as detection. Several of the other tests (Numbers 5,6, and 8) are used routinely in numerous protocols at our center and are good representatives of several basic classes of olfactory tests, including those of odor memory, discrimination, and suprathreshold scaling. ${ }^{1}$

1. University of Pennsylvania Smell Identification Test (UPSIT). This standardized, commercially available test is described in detail elsewhere (Doty, Frye, \& Agrawal, 1989; Doty et al., 1984). Briefly, a subject is required to identify, in a four-alternative multiple-choice format, each of 40 odorants presented on microencapsulated "scratch and sniff" labels. For example, one of the test items reads, "This odor smells most like: (a) chocolate; (b) banana; (c) onion; or (d) fruit punch." The subject must provide a response even if no odor is perceived (i.e., the test is forced choice). The number of items out of 40 that were answered correctly served as the dependent measure.

2. Single Ascending Series Butanol Odor Detection Threshold Test. The stimuli used in this standardized test, which is described by Cain, Gent, Goodspeed, and Leonard (1988) and Stevens, Cain, and Burke (1988), consist of 12 ternary aqueous dilution steps of $n$-butanol (from a $4 \%$ initial dilution mixture) presented in ascending order in a two-alternative forced-choice paradigm. The threshold was defined as the lowest concentration at which a subject correctly indicated which of two plastic squeeze bottles- one containing the odorant and the other the diluent-produced the stronger odor on five consecutive trials (see Cain \& Rabin, 1989).

3. Phenyl Ethyl Alcohol Single-Staircase Odor Detection Threshold Test. In this test, detection threshold values for the roselike odorant phenyl ethyl alcohol are determined by using a modified single-staircase procedure described in detail elsewhere (Deems \& Doty, 1987; Doty et al., 1984). In the present study, the staircase was begun at the $-6.50 \mathrm{log}$ concentration step of a half$\log$ step $(\mathrm{vol} / \mathrm{vol})$ dilution series extending from $-10.00 \mathrm{log}$ concentration to $-2.00 \log$ concentration. It was moved upward in full-log steps until correct detection occurred on five sets of consecutive trials at a given concentration. If an incorrect response was given on any trial, the staircase was moved upward a full-log step. When a correct response was made on all five trials, the staircase was reversed and subsequently moved up or down in half-log increments or decrements, depending upon the subject's performance on two pairs of trials (each pair consisting of a choice between a blank and an odorant) at each concentration step.The geometric mean of the first four staircase reversal points following the third staircase reversal was used as the threshold measure.

4. Single Series Phenyl Ethyl Methyl Ethyl Carbinol Odor Detection Threshold Test. This standardized test establishes a measure of detection threshold for the odorant phenyl ethyl methyl ethyl carbinol (PEMEC) by using a squeeze bottle procedure (see Amoore \& Ollman, 1983). The ascending method of limits odorant presentation procedure incorporates a dilution series analogous to that used in the $n$-butanol threshold test, except that only three correct pairs of trials at a given concentration are required to define the detection threshold value. The stimulus concentrations, which are not specified by the manufacturer, are reported in "decismels" in accordance with a $\log _{2}$ dilution sequence.

5. Odor Recognition Memory Test. On a given trial of this 12trial test, which is described in detail elsewhere (Bromley \& Doty, in press), a microencapsulated "target odorant" is presented to a subject. This is followed by four odorants from which the subject is instructed to select the one identical to the target stimulus. On four trials, a 10-sec interval is interspersed between the sampling of the target stimulus and the presentation of the first of the four alternatives. On four others, a 30-sec interval is enforced, whereas on the other four trials a $60-\mathrm{sec}$ period intervenes. The number of trials in which the target odor was correctly identified, irrespective of delay interval, served as the dependent measure.

6. Odor Discrimination Test. In this test, a subject is presented with 16 sets of three microencapsulated odorants (two same, one different) on separate pages of a cardboard test booklet (see Smith, Doty, Burlingame, \& McKeown, 1993). The stimuli on a given page of the test are presented in rapid succession and the examinee is asked to select the "odd" or "different" odor within each triad. The odorants of a triad were preselected to be equivalent in average perceived intensity, as determined from intensity ratings presented elsewhere (Doty et al., 1984). The number of triads in which the different stimulus was correctly reported served as the dependent measure.

7. Yes/No Odor Identification Test. This test utilizes $20 \mathrm{mi}-$ croencapsulated odorants from the UPSIT and is described in detail elsewhere (Corwin, 1989). Each of 20 odorants is presented twice-once with a descriptor that correctly describes the smell and once with a descriptor that does not. The subject's task is to report "yes" or "no" to whether the odor smells like the given descriptor. Two measures, derived from signal detection theory, served as dependent variables- $-d^{\prime}$ and $\mathrm{Cl}$ (Snodgrass \& Corwin, $1988) ; d^{\prime}$ reflects the sensory sensitivity and $\mathrm{Cl}$ reflects the response bias (i.e., the criterion an individual uses to make a decision about whether or not a stimulus is present).

8. Suprathreshold Amyl Acetate Odor Intensity and Odor Pleasantness Rating Test. In this test, which is similar in format to 
other tests in the literature (e.g., Lawless \& Malone, 1986), each subject was presented with $100-\mathrm{ml}$ glass sniff bottles containing different concentrations of amyl acetate $(-1.00,-2.00,-3.00$, and $-4.00 \log \mathrm{vol} / \mathrm{vol}$ ) diluted in USP grade light mineral oil. Each of the four stimuli was presented five times, in counterbalanced order, for a total of 20 trials. The subject was required to rate the perceived intensity and pleasantness of each stimulus on fully anchored, 9-point category scales (for intensity, 1 = no smell, $9=$ extremely strong; for pleasantness, $1=$ dislike extremely, $9=$ like extremely). Two measures were calculated for the odor intensity ratings: (1) the slope of the concentration/intensity function (following $\log$ transformation of the intensity ratings) obtained from a least squares linear regression analysis, and (2) the overall mean of the intensity ratings. For the pleasantness assessment, only the mean of the pleasantness ratings was used as the dependent measure, since (1) no single function that uniformly fit the response/concentration data for all subjects could be found, and (2) pleasantness ratings are relatively flat over a wide range of amyl acetate concent rations (Doty, 1975).

9. Toyota and Takagi (T\&T) Olfactometer. This nonforcedchoice standardized test, which is manufactured and used primarily in Japan, consists of five odorants, each diluted into eight logstep concentration series by using either propylene glycol or Nujol oil (for details, see Takagi, 1989; Yoshida, 1984). The stimuli are iso-valeric acid, skatole, $\beta$-phenyl ethyl alcohol, $\Gamma$-undecalactone, and methyl cyclopentenolone (cyclotene). The stimulus concentrations are presented in an ascending series and sniffed from strips of blotter paper dipped into the odorant solutions. The concentration at which a stimulus was first noticed (but usually not recognized) was defined as the detection threshold, and the concentration at which the subject could identify the odor was defined as the recognition threshold. The means of the detection and recognition threshold values of the subcomponents of the test were used as the dependent measures. ${ }^{2}$

\section{RESULTS}

\section{Correlations Among Test Measures}

Pearson correlation coefficients ( $r s$ ) were computed among the 13 test measures derived from the nine tests for the 97 subjects (Table 1). It is apparent from this table that many of the measures were relatively well correlated with one another, ${ }^{3}$ although notable exceptions occurred. For example, the mean odor pleasantness rating values and the response bias measure derived from the yes/no identification test were poorly correlated with all of the other measures. Similarly, the odor intensity rating measures (slope and mean) were poorly correlated with most of the other measures.

In an effort to mitigate possible contamination of the correlation matrix from differential influences of gender, age, smoking, and years of education on the test measures, a correlation matrix analogous to that in Table 1 was computed by using the residuals from a multiple regression analysis, in which these four variables served as independent variables and the 13 test measures served as dependent variables. The resulting pattern of correlations (Table 2) was similar to the pattern in Table 1, although in most cases smaller correlations were observed, reflecting, in part, attenuation in the range of test scores.

\section{Principal Component Analysis of Intercorrelation and Residual Matrices}

The intercorrelation matrix among the 13 test measures (Table 1) was subjected to principal components analysis, a procedure that, although similar to factor analysis, does not suffer from the factor indeterminacy of factor analysis (Wilkinson, 1990). Four principal components emerged with eigen values $>1.00$ (Table 3 ). The first evidenced loadings $>.40$ from 10 of the 13 test measures. The second was comprised of the primary loadings from two measures of suprathreshold odor intensity (i.e., the mean and the slope of the intensity ratings), whereas the third was comprised mainly of a strong primary loading from the mean of the suprathreshold odor pleasantness ratings. The fourth component was comprised of a primary loading from the response bias measure of the yes/no odor identification test. ${ }^{4}$

The correlation matrix of residuals (Table 2) for the 13 variables was also subjected to analysis. As can be seen in Table 4, a component structure pattern very similar to that observed in Table 3 appeared. The major differences were that the primary yes/no bias measure loaded on the second principal component rather than on the fourth (which gained a primary loading from the $T \& T$ detection threshold measure) and secondary or tertiary loadings from the mean and slope values of the odor intensity rating test were markedly reduced.

Table 1

Pearson Correlation Values Among the 13 Olfactory Measures Evaluated in the Study $(n=97)$

\begin{tabular}{|c|c|c|c|c|c|c|c|c|c|c|c|c|c|}
\hline & 1 & 2 & 3 & 4 & 5 & 6 & 7 & 8 & 9 & 10 & 11 & 12 & 13 \\
\hline 1. Butanol threshold & 1.00 & & & & & & & & & & & & \\
\hline 2. Odor discrimination & .43 & 1.00 & & & & & & & & & & & \\
\hline 3. Odor memory & .25 & .47 & 1.00 & & & & & & & & & & \\
\hline 4. Phenyl ethyl alcohol threshold & -.36 & -.47 & -.57 & 1.00 & & & & & & & & & \\
\hline 5. Phenyl ethyl methyl ethyl carbinol threshold & -.29 & -.41 & -.36 & .43 & 1.00 & & & & & & & & \\
\hline 6. Odor intensity rating (slope) & .41 & .26 & .19 & -.24 & -.18 & 1.00 & & & & & & & \\
\hline 7. Odor intensity rating (mean) & .08 & .22 & .35 & -.23 & -.33 & -.07 & 1.00 & & & & & & \\
\hline 8. Odor pleasantness rating (mean) & -.14 & -.02 & -.01 & .09 & -.03 & -.14 & -.13 & 1.00 & & & & & \\
\hline 9. T\&T identification (composite) & -.21 & -.40 & -.43 & .47 & .43 & -.12 & -.33 & .04 & 1.00 & & & & \\
\hline 10. T\&T detection (composite) & -.24 & -.33 & -.31 & .46 & .35 & .08 & -.38 & .04 & .59 & 1.00 & & & \\
\hline 11. University of Pennsylvania Smell Identification Test & .41 & .59 & .62 & -.63 & -.49 & .29 & .27 & -.05 & -.61 & -.41 & 1.00 & & \\
\hline 12. Yes/no discrimination $\left(d^{\prime}\right)$ & .39 & .45 & .49 & -.53 & -.44 & 17 & .30 & -.14 & -.47 &.- .34 & 60 & 1.00 & \\
\hline 13. Yes/no bias $(\mathrm{Cl})$ & .10 & -.13 & .03 & -.10 & -.02 & .12 & -.11 & -.01 & .09 & .05 & .00 & -.09 & 1.00 \\
\hline
\end{tabular}

Note-Correlations (Bonferroni corrected for inflated alpha) $\geq .35$ significant at $p<.05$. 
Table 2

Pearson Correlation Matrix Computed by Using the Residuals From a Multiple Regression Analysis in Which Gender, Age, Smoking, and Years of Education Served as Independent Variables and the 13 Test Measures as Dependent Variables

\begin{tabular}{|c|c|c|c|c|c|c|c|c|c|c|c|c|c|}
\hline & 1 & 2 & 3 & 4 & 5 & 6 & 7 & 8 & 9 & 10 & 11 & 12 & 13 \\
\hline 1. Butanol threshold & 1.00 & & & & & & & & & & & & \\
\hline 2. Odor discrimination & .30 & 1.00 & & & & & & & & & & & \\
\hline 3. Odor memory & .06 & .34 & 1.00 & & & & & & & & & & \\
\hline 4. Phenyl ethyl alcohol threshold & -.24 & -.34 & -.42 & 1.00 & & & & & & & & & \\
\hline 5. Phenyl ethyl methyl ethyl carbinol threshold & -.06 & -.26 & -.14 & .24 & 1.00 & & & & & & & & \\
\hline 6. Odor intensity rating (slope) & .32 & .10 & .01 & -.13 & .03 & 1.00 & & & & & & & \\
\hline 7. Odor intensity rating (mean) & -.12 & .01 & .19 & .01 & -.05 & -.30 & 1.00 & & & & & & \\
\hline 8. Odor pleasantness rating (mean) & -.11 & .02 & .04 & .05 & -.12 & -.12 & -.11 & 1.00 & & & & & \\
\hline 9. $T \& T$ identification (composite) & -.05 & -.22 & -.27 & .25 & .28 & .00 & -.14 & -.01 & 1.00 & & & & \\
\hline 10. T\&T detection (composite) & -.04 & -.08 & -.06 & .16 & .07 & .32 & -.17 & .00 & .38 & 1.00 & & & \\
\hline 11. University of Pennsylvania Smell Identification Test & .29 & .45 & .49 & -.48 & -.30 & .15 & .01 & .02 & -.45 & -.07 & 1.00 & & \\
\hline 12. Yes/no discrimination $\left(d^{\prime}\right)$ & .29 & .38 & .41 & -.43 & -.28 & .08 & .09 & -.10 & -.33 & -.12 & .54 & 1.00 & \\
\hline 13. Yes/no bias (Cl) & .16 & -.05 & -.09 & -.17 & -.23 & .22 & .02 & -.03 & .03 & -.13 & .15 & -.12 & 1.00 \\
\hline
\end{tabular}

Note-C Correlations (Bonferroni corrected for inflated alpha) $\geq .35$ significant at $p<.05$.

\section{DISCUSSION}

The present findings, while in accord with earlier observations of correlations among threshold tests and odor identification tests in a number of normal and patient groups (e.g., Cain et al., 1988; Cain \& Rabin, 1989; Deems et al., 1991; Doty, Deems, \& Stellar, 1988; Doty, Reyes \& Gregor, 1987; Doty et al., 1984; Yoshida, 1984), reflect an expansion of the concept of commonality to a wide range of nominally distinct olfactory tests. These findings suggest that many olfactory tests, for all practical purposes, measure a common source of variance, perhaps analogous to the " $G$ " factor observed in intelligence measurement theory (Spearman, 1904).

It is not known what this source of variance represents. One possibility is that olfactory perception is multidimensional and that a variety of olfactory tests tap, to a large degree, elements that are defined by most of the tests evaluated in this work (e.g., odor memory, discrimination, detection, and identification). From this perspective, even the act of detecting an odorant can be viewed as requiring some degree of ability to remember the odorant and to discriminate it from a blank; however, the elements would have to interrelate in such a way that they would not appear as separate components in the principal components analysis. At the other extreme, many of the olfactory tests evaluated in this study may depend disproportionately upon a single element (e.g., stimulus encoding processes at the receptor sheet). This element might, in turn, determine the degree to which the tests are interrelated. For example, damage to the olfactory receptor cells within the neuroepithelium would be expected to have an impact on a wide variety of olfactory test measures, particularly those that incorporate weak or moderately intense odorants. Importantly, interindividual differences in test scores, which are essential to the production of correlations among test measures, may depend upon the relative degree of such damage. It is well established, for example, that the olfactory neuroepithelium, unlike more central olfactory centers, undergoes considerable deterioration throughout the normal life span (see Curcio, McNelly, \& Hinds, 1985; Nakashima, Kimmelman,

Table 3

Component Loadings for the Principal Component Analysis Performed on the Intercorrelation Matrix Among the Olfactory Test Measures of the Study

\begin{tabular}{|c|c|c|c|c|}
\hline & 1 & 2 & 3 & 4 \\
\hline University of Pennsylvania Smell Identification Test & .85 & .07 & -.11 & .06 \\
\hline Phenyl ethyl alcohol threshold & -.77 & -.09 & .10 & .16 \\
\hline Yes/no $\left(d^{\prime}\right)$ & .74 & .00 & .12 & .10 \\
\hline T\&T identification (composite) & -.72 & .28 & .03 & .05 \\
\hline Odor memory & .72 & -.02 & -.13 & -.04 \\
\hline Odor discrimination & .71 & .11 & -.06 & .34 \\
\hline Phenyl ethyl methyl ethyl carbinol threshold & -.65 & .06 & .15 & .05 \\
\hline T\&T detection (composite) & -.61 & .41 & -.02 & .24 \\
\hline Butanol threshold & .54 & .50 & .10 & .09 \\
\hline Odor intensity rating (mean) & .46 & -.47 & .30 & -.21 \\
\hline Odor intensity rating (slope) & .32 & .72 & .07 & .17 \\
\hline Odor pleasantness rating (mean) & -.12 & -.20 & -.90 & .22 \\
\hline Yes/no (bias) & -.02 & .45 & -.28 & -.81 \\
\hline Percentage of total variance accounted for & 37.20 & 11.66 & 8.25 & 7.74 \\
\hline
\end{tabular}

Note-Loadings $>.40$ are considered salient. Primary loadings are in boldface for emphasis (see note 4). 
Table 4

Component Loadings for the Principal Component Analysis Performed on the Correlation Matrix by Using the Residuals from a Multiple Regression Analysis in Which Gender, Age, Smoking, and Years of Education Served as Independent Variables and the 13 Test Measures as Dependent Variables

\begin{tabular}{lrrrr}
\hline & 1 & \multicolumn{1}{c}{ 2 } & \multicolumn{1}{c}{3} & \multicolumn{1}{c}{4} \\
\hline University of Pennsylvania Smell Identification Test &. $\mathbf{8 5}$ & .07 & -.11 & .06 \\
Yes/no ( $d^{\prime}$ ) & -.77 & -.09 & .10 & .16 \\
Phenyl ethyl alcohol threshold & .74 & .00 & .12 & .10 \\
Odor discrimination & .71 & -.11 & .05 & -.05 \\
Odor memory & .64 & -.12 & .43 & .26 \\
T\&T identification (composite) & -.60 & .33 & .10 & -.01 \\
Butanol threshold & .51 & .37 & -.21 & -.46 \\
Phenyl ethyl methyl ethyl carbinol threshold & -.50 & .04 & .49 & -.31 \\
Odor intensity rating (slope) & .17 & .75 & .28 & .05 \\
Yes/no (bias) & .30 & .59 & -.35 & -.15 \\
Odor intensity rating (mean) & .24 & -.58 & .20 & -.23 \\
Odor pleasantness rating (mean) & .02 & -.21 & -.59 & .51 \\
T\&T detection (composite) & -.40 & .34 & .31 &. $\mathbf{6 2}$ \\
Percentage of total variance accounted for & 30.13 & 13.46 & 9.64 & 9.03 \\
\hline
\end{tabular}

Note-Loadings $>, 40$ are considered salient. Primary loadings are in boldface for emphasis (see note 4).

\& Snow, 1984), being susceptible to insults from environmental agents (including viruses and airborne toxins), trauma, and a variety of disease processes (Deems et al., 1991; Jafek, Eller, Esses, \& Moran, 1989; Moran, Jafek, Rowley, \& Eller, 1985; Trojanowski, Newman, Hill, \& Lee, 1991).

The fact that the suprathreshold intensity and pleasantness ratings, as well as the response bias measure from the yes/no odor identification test, exhibited their primary loadings on components other than the first one suggests that these tests may be less sensitive to sensory processes sampled by most of the other tests. It is not surprising that the response bias measure is largely independent of such processes, and there is precedence for the independence of a hedonic dimension from other dimensions of olfactory perception in persons who do not exhibit anosmia or marked hyposmia. Thus, a unique hedonic dimension is often observed in multidimensional scaling studies (e.g., Schiffman, 1974) and a wide variety of odor classification schemes view such a dimension as an independent sensory attribute (see Harper, Bate Smith, \& Land, 1968; Moncrieff, 1966). However, the apparent partial or near-total independence of the suprathreshold scaling measures from the other types of olfactory test measures (particularly in the case of the residuals; see Table 4) is somewhat difficult to explain. Nevertheless, this phenomenon may have a parallel in auditory and gustatory psychophysics, in that "recruitment" of additional sensory fibers may occur at higher stimulus intensities, thereby producing suprathreshold intensity functions that appear normal (see, e.g., Bartoshuk, Rifkin, Marks, \& Bars, 1986). In the present work, such recruitment could include fibers from free nerve endings of the trigeminal nerve that are distributed throughout the nasal mucosa, since amyl acetate is known to have trigeminal stimulative properties at higher concentrations and receives intensity ratings from anosmics similar to those observed in normosmics
(Doty et al., 1978). Such a notion may explain why some suprathreshold scaling parameters are insensitive to changes in the ability to smell related to age and Alzheimer's disease (e.g., Green et al., 1989; Rovee, Cohen, \& Shlapack, 1975).

Regardless of the physiologic basis of the present findings, if, in fact, the suprathreshold intensity and hedonic scaling measures are largely independent of the other measures, they may prove to be useful additions to a comprehensive chemosensory test battery, such as those employed in clinical settings. However, more data are needed to verify this point, since one cannot assume that simply because a test fails to correlate with other sensory tests it will serve as a useful index of sensory function. In the case of gustation, Bartoshuk (1978) has noted that suprathreshold magnitude estimation tests of taste intensity are often unrelated to detection threshold measures, and points out that suprathreshold intensity functions can vary independently of the taste threshold. The present data suggest that this may also be the case with the sense of smell.

The degree to which the component structure found in the present study is generalizable to other subject groups, such as those with focal brain disorders or lesions that involve cortical regions or pathways associated with olfactory function, is not clear. Although some investigators report that persons with brain damage secondary to Korsakoff's psychosis or removal of sectors of the cerebral cortex to control intractable seizure activity evidence problems in odor identification, but not in odor detection (Jones-Gotman \& Zatorre, 1988; Mair et al., 1986), other investigators observe changes in a broad array of test measures in such patients, including measures of odor identification and detection (Martinez et al., 1993; Potter \& Butters, 1980; West \& Doty, in press). Clearly, more research is needed to ascertain the generality of the present component structure. 
The correlations observed between the tests evaluated in this study are different, in some cases, from those observed in earlier studies, conceivably reflecting parameters such as the differences in the types of subjects examined and their experience in sensory evaluation. Thus, Doty et al. (1984) previously reported a -.79 correlation between the UPSIT and phenyl ethyl alcohol thresholds in a group of subjects that included individuals known to have olfactory dysfunction, and Cain and Rabin (1989) reported a .76 correlation between the UPSIT and an $n$-butanol threshold determined by a single ascending series in a similar group of subjects. In the present study, however, both of these relationships were considerably lower (respective $r s=-.63$ and .41 ), possibly reflecting the lack of inclusion of persons with known chemosensory abnormalities (i.e., the presence of a more restricted distribution of scores). Similarly, Cain and Gent (1991) reported, in 32 subjects, a - .66 correlation coefficient between butanol threshold values and PEMEC threshold values using the procedures employed in the present study. The correlation observed in our study between these two variables $(r=-.29)$ is considerably lower than this value. The reason for this difference is not known.

In conclusion, the present study demonstrates that a number of nominally distinct olfactory tests measure a common source of variance in subjects spanning a wide age range, and that some tests, such as the suprathreshold odor intensity and pleasantness rating tests, may sample somewhat different components of variance. Future research is needed to determine whether the relationships observed in the present study, as evidenced in the component structure of the principal component analyses, generalize to other subject populations. In addition, more work is needed on the development of olfactory tests that challenge central cognitive processing (e.g., odor reversal learning) in an effort to add sophistication to our ability to detect subtle aspects of central olfactory system function.

\section{REFERENCES}

Amoore, J. E., Ollman, B. G. (1983). Practical test kits for quantitatively evaluating the sense of smell. Rhinology, 21, 49-54.

BartoshuK, L. M. (1978). The psychophysics of taste. American Journal of Clinical Nutrition, 31, 1068-1077.

Bartoshuk, L. M., Rifkin, B., Marks, L. E., \& Bars, P. (1986). Taste and aging. Journal of Gerontology, 41, 51-57.

BRoMLEY, S. M., \& Doty, R. L. (in press). Odor recognition memory is better under bilateral than unilateral test conditions. Cortex.

CAIN, W. S. (1978). History of research on smell. In E. C. Carterette \& M. P. Friedman, Handbook of perception (pp. 197-229). New York: Academic Press.

CAIN, W. S., \& GENT, J. F. (1991). Olfactory sensitivity: Reliability, generality, and association with aging. Journal of Experimental Psychology: Human Perception \& Performance, 17, 382-391.

Cain, W. S., Gent, J. F., Goodspeed, R. B., \& Leonard, G. (1988). Evaluation of olfactory dysfunction in the Connecticut Chemosensory Clinical Research Center. Laryngoscope, 98, 83-88.

CAIN, W. S., \& RABIN, R. D. (1989). Comparability of two tests of olfactory functioning. Chemical Senses, 14, 479-485.

CoRwin, J. (1989). Olfactory identification in hemodialysis: Acute and chronic effects on discrimination and response bias. Neuropsychologia, 27, 513-522.

Curcio, C. A., MCNelly, N. A., \& Hinds, J. W. (1985). Aging in the rat olfactory system: Relative stability of piriform cortex contrasts with changes in olfactory bulb and olfactory epithelium. Journal of Comparative Neurology, 235, 519-528.

DeEms, D. A., \& Doty, R. L. (1987). Age-related changes in the phenyl ethyl alcohol odor detection threshold. Transactions of the Pennsylvania Academy of Ophthalmology \& Otolaryngology, 39, 646-650.

Deems, D. A., Doty, R. L., Settle, R. G., Moore-Gillon, V., Shaman, P., Mester, A. F., Kimmelman, C. P., Brightman, V. J., \& SNOW, J. B., JR. (1991), Smell and taste disorders: A study of 750 patients from the University of Pennsylvania Smell and Taste Center (1981-1986). Archives of Otolaryngology Head \& Neck Surgery, 117, 519-528.

Doty, R. L. (1975). An examination of relationships between the pleasantness, intensity, and concentration of 10 odorous stimuli. Perception \& Psychophysics, 17, 492-496.

DotY, R. L. (1991). Olfactory system. In T. V. Getchell, R. L. Doty, L. M. Bartoshuk, \& J. B. Snow, Jr. (Eds.), Smell and taste in health and disease (pp. 175-203). New York: Raven.

Doty, R. L. (1992). Diagnostic tests and assessment. Journal of Head Trauma, 7, 47-65.

Doty, R. L., Brugger, W. E., Jurs, P. C., OrndorfF, M. A., SNyder, P. J., \& LoWRY, L. D. (1978). Intranasal trigeminal stimulation from odorous volatiles: Psychometric responses from anosmic and normal humans. Physiology \& Behavior, 20, 175-185.

Doty, R. L., DeEMS, D., \& STELlaA, S. (1988). Olfactory dysfunction in Parkinson's disease: A general deficit unrelated to neurologic signs, disease stage, or disease duration. Neurology, 38, 1237-1244.

Doty, R. L., FrYE, R. E., \& Agrawal, U. (1989). Internal consistency reliability of the fractionated and whole University of Pennsylvania Smell Identification Test. Perception \& Psychophysics, 45, 381-384.

Doty, R. L., ReYes, P., \& Gregor, T. (1987). Presence of both odor identification and detection deficits in Alzheimer's disease. Brain Research Bulletin, 18, 597-600.

Doty, R. L., Shaman, P., \& Dann, M. (1984). Development of the University of Pennsylvania Smell Identification Test: A standardized microencapsulated test of olfactory function. Physiology \& Behavior, 32, 489-502.

Engen, T. (1983). The perception of odors. New York: Academic Press.

GorsuCH, R. L. (1983). Factor analysis. Hillsdale, NJ: Erlbaum.

Green, J. E., Songsanand, P., Peretz, S., Hsu, P., Corkin, S., \& Growden, J. H. (1989). Dissociation between basic and high order olfactory capacities in Alzheimer's disease. In R. J. Wurtman, S. H. Corkin, J. H. Growden, \& E. Ritter-Walker (Eds.), Proceedings of the Fifth Meeting of the International Study Group on Pharmacological Memory Disorders Associated with Aging (pp. 449-455). Cambridge, MA: Center for Brain Sciences and Metabolism Charitable Trust.

GuILFORd, J. P. (1954). Psychometric methods. New York: McGrawHill.

Harper, R., Bate Smith, E. C., \& Land, D. G. (1968). Odour description and odour classification. New York: Elsevier.

JafeK, B. W., Eller, P. M., Esses, B. A., Moran, D. T. (1989). Posttraumatic anosmia: Ultrastructural correlates. Archives of Neurology, 46, 300-304.

Jones-GotMAN, M., \& ZATORRE, R. J. (1988). Olfactory identification deficits in patients with focal cerebral excision. Neuropsychologia, 26, 387-400.

KösTER, E. P. (1975). Human psychophysics in olfaction. In D. G. Moulton, A. Turk, \& J. W. Johnston, Jr. (Eds.), Methods in olfactory research (pp. 345-374). New York: Academic Press.

LAWLESS, H. T., \& MAlONE, G. J. (1986). The discriminative efficiency of common scaling methods. Journal of Sensory Studies, 1 , 85-98.

Mair, R. G., DơTy, R. L., Kelly, K. M., Wilson, C. S., Langlais, P. J., MCENTEe, W. J., \& VollmeCKE, T. A. (1986). Multimodal sensory discrimination deficits in Korsakoff's psychosis. Neuropsychologia, 24, 831-839. 
Martinez, B. A., Cain, W. S., De WiJk, R. A., Spencer, D. D., NovELLY, R. A., \& SAAS, K. J. (1993). Olfactory functioning before and after temporal lobe resection for intractable seizures. Neuropsychology, 7, 351-363.

MONCRIEFF, R. W. (1966). Odour preferences. New York: Wiley.

Moran, D. T., JafeK, B. W., Rowley, J. C., \& Eller, P. M. (1985). Electron microscopy of olfactory epithelia in two patients with anosmia. Archives of Otolaryngology, 111, 122-126.

Nakashima, T., Kimmelman, C. P., \& SNow, J. B., JR. (1984). Structure of human fetal and adult olfactory neuroepithelium. Archives of Otolaryngology, 110, 641-646.

POTTER, H., \& BUTTERs, N. (1980). An assessment of olfactory deficits in patients with damage to prefrontal cortex. Neuropsychologia, 18, 621-628.

Rovee, C. K., Cohen, R. Y., \& Shlapack, W. (1975). Life span stability in olfactory sensitivity. Developmental Psychology, 11, 311318.

Rozeвоom, W. W. (1982). The determinacy of common factors in large item domains. Psychometrika, 47, 281-295.

SChIffMan, S. S. (1974). Physicochemical correlates of olfactory quality. Science, $185,112-117$.

Smith, R. S., Doty, R. L., Burlingame, G. K., \& McKeown, D. A. (1993). Smell and taste function in the visually impaired. Perception \& Psychophysics, 54, 649-655.

SnOdgrass, J. C., \& CoRwin, J. (1988). Pragmatics of measuring recognition memory: Applications to dementia and amnesia. Journal of Experimental Psychology: General, 117, 34-50.

SPEARMAN, C. (1904). "General intelligence," objectively determined and measured. American Journal of Psychology, 15, 201-293.

Stevens, J. C., CaIN, W. S., \& Burke, R. J. (1988). Variability of olfactory thresholds. Chemical Senses, 13, 643-653.

TAKAGI, S. F. (1989). Human olfaction. Tokyo: University of Tokyo Press.

Trojanowski, J. Q., Newman, P. D., Hill, W. D., \& Lee, V. M.-Y. (1991). Human olfactory epithelium in normal aging, Alzheimer's disease, and other degenerative disorders. Journal of Comparative Neurology, 310, 365-376.

VALENTIn, G. (1848). Lehrbuch der Physiologie des Menschen. Braunschweig.

Vollmecke, T. A., \& Doty, R. L. (1985). Development of the Picture Identification Test (PIT): A research companion to the University of Pennsylvania Smell Identification Test. Chemical Senses, 10, 413-414.

WENZEL, W. (1948). Techniques in olfactometry. Psychological Bulletin, 45, 231-246.

WeST, S. E., \& DorY, R. L. (in press). The influence of epilepsy and temporal lobe resection on olfactory function: A review. Epilepsia.

WhIPPLE, G. M. (1914). Manual of mental and physical tests. Baltimore, MD: Warwick \& York.

WILKINSON, L. (1990). SYSTAT: The system for statistics. Evanston, IL: SYSTAT, Inc.

Yoshida, M. (1984). Correlation analysis of detection threshold data for "standard test" odors. Bulletin of the Faculty of Science \& Engineering of Chuo University, 27, 343-353.

\section{NOTES}

1. It should be noted that the tests evaluated in our study likely account for the vast majority of olfactory tests administered in the world. Test 1 is available commercially and is currently used in approximately 3,000 clinics in North America. This test has been administered to at least 35,000 persons over the last decade. Test 2 is used routinely at the University of Connecticut Clinical Chemosensory Research Center, and Test 3 is used at the University of Pennsylvania Smell and Taste Center in clinical and experimental applications. Test 4 is also available commercially; it is used largely in industrial settings. Tests 5,6 , and 8 are routinely used at our center. Test 9 , which is manufactured in Japan, is the only olfactory test in Japan for which physicians can obtain insurance reimbursement. Next to the UPSIT, it is probably the most widely administered olfactory test.

2. Collapsing the $T \& T$ olfactometer measures into single identification and detection threshold values increased the reliability of the $\mathrm{T} \& \mathrm{~T}$ measure and allowed us to maintain, in the subsequent principal components analysis, the variable-to-subject ratio at the recommended minimum of $5: 1$ (see Gorsuch, 1983, p. 332). We also explored the influences of various transformations on the intercorrelations among the variables of the data set. Since most studies employ untransformed values as their measures, and since such transformations did not alter the obtained findings in meaningful ways, the data from only the untransformed variables are presented here.

3. The differences in sign of the $r$ values reflect scaling factors and in this context should be ignored.

4. According to Gorsuch (1983, p. 208), the lower bound for the size of a salient component or factor loading can be calculated by doubling the minimum significant correlation for a given sample size. For example, a sample size of 100 requires loadings $>.40$ to be considered salient at the $.05 \alpha$ level. When we performed a jackknife procedure on the residuals in which we randomly omitted 10 or 20 subjects, four components were retained with eigen values $>1.00$. The pattern of loadings on these components were analogous to those obtained from the full 97 subjects. When we performed a similar procedure on the raw correlation matrix, only three components had eigen values $>1.00$ (the fourth had an eigen value of .99). However, similar general patterns of loadings occurred even in the three-component solution. Thus, when 10 and 20 subjects were randomly omitted, all but three of the variables had their primary loadings on the first principal component. The second component consisted of the primary loading from the odor intensity rating slope, whereas the third component consisted of primary loadings from the mean odor pleasantness rating and from the yes/no bias measure. Thus, the general component structure described in this study is reasonably stable.

(Manuscript received May 28, 1993; revision accepted for publication June 28,1994 ). 